\title{
Narrativas de mujeres que transitan la experiencia de la "locura". Un recorrido por los itinerarios terapéuticos en Salud Mental ${ }^{1}$
}

\author{
Liza María Benedetti², María Kendziur ${ }^{3}$
}

\begin{abstract}
El presente trabajo tiene como finalidad socializar y poner en debate algunos de los hallazgos obtenidos en un proceso de investigación desarrollado en la intersección de los campos de la Salud Mental y el Género. Partimos por considerar y vincular tres conceptos centrales: narrativas, experiencia y significados, que a lo largo de toda la producción fuimos articulando con otros conceptos que se desprenden de nuestro planteo inicial. El objetivo es recuperar los significados que las mujeres usuarias de Servicios de Salud Mental otorgan a la experiencia de la "locura" y conocer los relatos respecto de los itinerarios terapéuticos recorridos. Con el propósito de trabajar en clave de "narrativas", esta investigación se inscribe dentro de la lógica cualitativa, en tanto supone un esfuerzo por generar conocimiento de la realidad social desde el punto de vista de quienes la producen, la viven y la significan.
\end{abstract}

Palabras clave: Salud Mental, género, narrativas, itinerarios terapéuticos

\section{Relatos de mulheres que transitam a experiência da "loucura". Um percurso pelos itinerários terapêuticos em Saúde Mental*}

\section{RESUMO}

O presente trabalho tem como finalidade socializar e lançar ao debate algumas das descobertas obtidas num processo de investigação desenvolvido na intersecção dos campos de Saúde Mental e Gênero. Começamos considerando e vinculando três conceitos centrais: relatos, experiências e significados, que durante toda a produção fomos articulando com outros conceitos que decorrem da nossa abordagem inicial. O objetivo é recuperar os significados que as mulheres usuárias de Serviços de Saúde Mental

1 Artículo recibido: 30/05/2016. Artículo aprobado: 26/09/2016.

2 Argentina. Trabajadora Social. Universidad Nacional de Entre Ríos. Correo electrónico: lizabenedettimochi@hotmail.com

3 Argentina. Trabajadora Social. Universidad Nacional de Entre Ríos. Correo electrónico: mariakendziur@hotmail.com 
conferem à experiência da "loucura" e conhecer os relatos em relação aos itinerários terapêuticos percorridos. Com o propósito de trabalhar em termos de "narrativas", esta investigação se encaixa dentro da lógica qualitativa, porquanto supõe um esforço por gerar conhecimento da realidade social desde o ponto de vista dos que a produzem, vivem e significam.

Palavras-chave: saúde mental, gênero, relatos, itinerários terapêuticos

\title{
Narratives of women who undergo the experience of "madness". A tour through the therapeutic itineraries in Mental Health
}

\begin{abstract}
The present work aims to socialize and bring to discussion some of the findings acquired during a process of research in the fields of Mental Health and Gender. We set from considering and linking three central concepts: narratives, experience and significations; through our production, we articulate them as well with other concepts that arise from our initial statements. Our objective is to recover the significations that women users of Mental Health Services give to the experience of "madness", and to know their accounts concerning the therapeutic itineraries traversed. In the light of being able to work in a narrative key, this investigation enrolls into the qualitative research logic, insofar as it represents an effort for the generation of knowledge of the social reality from the view point of those who produce, live and signify it.
\end{abstract}

Keywords: Mental Health, gender, narratives, therapeutic itineraries

\section{Introducción}

Las reflexiones presentadas en el siguiente trabajo se corresponden con los hallazgos obtenidos a partir de la realización de una investigación denominada "Narrativas de Mujeres que transitan la experiencia de la "Locura". Un recorrido por los Itinerarios terapéuticos en Salud Mental”, realizada durante los años 2012-2013. Para la misma se trabajó con siete mujeres usuarias de Hospitales monovalentes de Salud Mental, de la ciudad de Paraná [Entre Ríos] y de Santa Fe [Santa Fe].

Dicha investigación, en la cual fue transversal un análisis desde la perspectiva de Género, tuvo como apuesta fundamental poder aportar al campo disciplinar del Trabajo Social con la generación de conocimientos acerca de los complejos procesos que 
se producen en la relación dialéctica entre el lugar que se les asigna a las mujeres en los procesos de salud-enfermedad-cuidados y el lugar que ellas ocupan, donde intervienen diversos actores entre los cuales ubicamos profesionales de nuestra disciplina.

La producción de un conocimiento desde las perspectivas escogidas contiene como posibilidad el llevar adelante un proceso de ruptura con aquellas prácticas y discursos de profesionales del Campo de la Salud, que históricamente han puesto en un lugar de estigmatización, como expresión de construcciones históricosociales, a la mujer y la locura.

El proceso investigativo permitió indagar sobre un escenario de intervención complejo, donde se desarrollan prácticas discriminatorias que se constituyen en una fuente sustancial de producción y reproducción de malestares, vinculados los mismos a las particularidades que se advierten al trabajar sobre problemáticas que se encuentran atravesadas por el género, la pobreza y el sufrimiento psíquico.

Nuestro interés radicó, entonces, en poder retomar las voces de las propias mujeres, poder interpretar cómo estas significan sus experiencias, queriendo con esto aportar un conocimiento en el que la fuente empírica principal sea la palabra del actor. En este sentido, es que nuestra apuesta metodológica tuvo sus raíces en las narrativas de las mismas, sabiendo que "la narración autobiográfica no es para indagar en la parte íntima de una vida, sino para acceder a un discurso construido en un contexto de significado, objetivado en el lenguaje" (Lindón, 1999, p. 299).

Consideramos que el conocimiento producido desde esta perspectiva se constituye en un elemento de suma riqueza para el Trabajo Social; tal como expresa Rotondi (2003), “[...] No pretendemos, obviamente, decir que es suficiente, pero sí creemos que es altamente eficiente profundizar en la mirada de los sujetos para poder darle vida al diseño de nuestras estrategias de trabajo" (Rotondi, citado en Aquín, 2003). 
Para esta presentación se expondrán algunos hallazgos que permiten situar a los sujetos/actores de la investigación/intervención desde un lugar activo, protagonista, buscando desde allí nutrir al campo disciplinar a partir de una perspectiva que reconozca el lugar que efectivamente las mujeres ocupan en su proceso de saludenfermedad-cuidados. Previo a ello y para facilitar la comprensión del lector acerca de la propuesta, nos referiremos de manera breve al planteamiento categórico/conceptual desde donde fue realizada la indagación y las decisiones metodológicas que fueron asumidas. Para, finalmente, exponer algunas de las conclusiones que, como propuestas a futuro, resultan ejes significativos y ricos para el debate al interior del campo.

\section{Algunas claves para la lectura: planteamiento categórico- conceptual}

en orden a poder producir un conocimiento centrado en los relatos de las mujeres entrevistadas, se partió por considerar y vincular tres conceptos centrales: narrativas, experiencia y significados, los cuales fueron utilizados de manera permanente en articulación con otras categorías. El trabajo con narrativas posibilitó describir y desentrañar el proceso de constitución y re-creación de sentidos que otorgan los sujetos a sus propias prácticas, inscriptas en diferentes contextos y sobre la base de la interpretación de sus saberes, ideas, creencias, valores o, en otras palabras, de su subjetividad; dicha decisión teórica-metodológica se correspondió con el propósito principal de recuperar e indagar en los significados que las mujeres usuarias de Servicios de Salud Mental otorgan a su propia experiencia vinculada al padecimiento subjetivo.

Lindón (1999) define tres rasgos característicos de la noción de Narrativas. El primero de ellos refiere a que los relatos autobiográficos poseen un carácter experiencial; en el relato el narrador significa, cuenta, recuerda y conecta anécdotas donde aparecen otros actores, pero son siempre experiencias de quien habla, convirtiéndose así el narrador en el personaje central. Un segundo rasgo las ubica como relatos, lo que supone pensar que el narrador le da una 
estructura propia a su narración. En tercer y último lugar, la autora nos invita a pensar en los relatos autobiográficos como significativos socialmente. "[...] Esto supone que al escoger y articular las vivencias para narrarlas de manera comprensible a los otros, el narrador recurre a su memoria y también a su contexto sociocultural" (Lindón, 1999, p. 299).

Otro aporte interesante lo realiza Ricoeur (2002), el cual refiere a las Narrativas como una "particular forma de reconstrucción de la experiencia, por la que, mediante un proceso reflexivo, se da significado a lo sucedido o vivido" (Ricoeur, citado en Bolívar Botía, 2002).

La categoría Experiencia, por su parte, es siempre una construcción subjetiva, inscripta en un contexto particular, narrada y construida por el sujeto que la vive. Así, poder recuperar la experiencia, no ya como un concepto acabado y cerrado, nos invita a dialogar con el par experiencias/significados que en la investigación se propuso conocer a través de las narrativas de mujeres. "El significado, entonces, es una cierta manera de dirigir la mirada hacia un aspecto de una vivencia que es propia y singular" (Badano, 2009, p. 117).

Cabe destacar que la opción de trabajar con los significados que construyen las mujeres acerca de sus experiencias en los procesos de salud -enfermedad- cuidados imbricados en el Campo de la Salud Mental, buscó poder comprender cómo estas hacen jugar en sus relatos diferentes actores, espacios y tiempos, poniendo en lugares protagónicos algunos sucesos y olvidando otros, en fin, construyendo un relato singular, propio, único. Ahora bien, es menester indicar que el trabajo con narrativas requiere de una comprensión del contexto más amplio donde se circunscriben los relatos, lo cual, sumado al interés por realizar una lectura desde la perspectiva de género, exigió para el proceso investigativo el observar los múltiples atravesamientos que transversalizan el Campo.

Sobre ello, se parte por advertir que cada práctica en salud construye un sujeto determinado, al cual le destina un tipo de inter- 
vención particular. En esta construcción se condensan y mezclan diversos paradigmas, culturas y modos de percepción del otro que dotarán a éste de un conjunto de atributos que pueden potenciarlo o estigmatizarlo, lo que influye en los diversos procesos de configuración de subjetividades particulares.

Para la realización de la investigación se trabajó con el concepto de subjetividad, propuesto desde la Psicología Social por González Rey (2011), desde el cual

[...] la idea de subjetividad permite integrar tanto al sujeto como a los diferentes espacios intersubjetivos, de forma que abre, en sus diferentes espacios de relación, la multiplicidad de formas que lo social, en todas sus expresiones indirectas, consecuencias, y retazos, toman los diferentes sistemas humanos de relación [...] (González Rey, 2011: 48).

Sobre esto, interesó particularmente conocer los relatos de mujeres que transitan la experiencia de la locura, a partir de considerar las particularidades que representan las subjetividades femeninas, entendiendo que "la subjetividad de la mujer es la particular e individual concepción del mundo y de la vida que cada una elabora a partir de su concepción genérica, de las adscripciones socioculturales que la cruzan [...]" (Rojas Ortíz, 2009, p. 3). Allí, se entretejen elementos, ideas, supuestos y prácticas de carácter socio-histórico que se internalizan, así como también, aspectos de la experiencia vivida por cada mujer en su tránsito por la locura, donde se construyen itinerarios terapéuticos singulares.

Consideramos, en este sentido, que el trabajo con los significados que las usuarias asignan a su experiencia cotidiana habilita un conocimiento particular para intervenir con mujeres, sabiendo que sus demandas deben comprenderse en relación a su socialización y a su modo particular de estar en el mundo.

Tomando los aportes de Mabel Burín (1987) y situándonos específicamente en la constitución de la subjetividad femenina y en las 
múltiples formas de malestar ligadas a la socialización genérica, debemos detenernos en ciertas particularidades que se corresponden con la existencia de determinadas condiciones históricas, sociales, culturales, jurídicas, etc., de existencia, bajo las cuales una mujer puede constituir su subjetividad. En esta línea, la ideología patriarcal es entendida como condición de realización de todas las prácticas que constituyen la práctica social, que a su vez, como plantea la autora, se encuentran sujetas a ella.

Si bien las prácticas sociales son el resultado de la acción de los sujetos, debemos reconocer, a la vez, que esos sujetos son hechos por -son efectos de- tales prácticas. La ideología patriarcal posiciona a las mujeres como sujetos dentro de las prácticas sociales de la maternidad y, por extensión, normativiza el deseo sexual femenino bajo la forma de deseo maternal, y del trabajo femenino como trabajo maternal y doméstico (Burín, 1987, p. 50).

Pese a lo dicho y continuando con los aportes de la autora mencionada, cabe destacar que estas subjetividades producidas no quedan sometidas de forma pasiva a aquellas estructuras donde fueron moldeadas, sino que los sujetos oponen resistencias que tienen que ver con reguladores de la Salud Mental. Este último elemento se constituyó en un punto de interés destacable durante el proceso de investigación en orden a poder dilucidar el inter- juego y puja constante que existe entre los usuarios/usuarias de los servicios de atención de Salud Mental y los estereotipos de género, los cuales producen y reproducen formas esperadas de estar, ver, sentir y actuar.

Dos últimas precisiones son necesarias de plantear aquí, en tanto se trata de conceptos contenedores de todo el proceso de la investigación: las nociones de Salud Mental y el Género; sobre este último, si bien existen muchas acepciones, para esta investigación la perspectiva adoptada puso el foco sobre las atribuciones socio-histórico-culturales que se depositan en cada sexo biológico, a partir de los cuales se conforman esperables que no son más que concepciones y representaciones que circulan en el 
imaginario social, que establecen modos adecuados de ser, coartando potencialidades, estigmatizando conductas diferentes y contribuyendo a la producción y reproducción de prácticas discriminatorias.

Dentro del género como perspectiva nos posicionamos al interior de los denominados "Estudios de la Mujer" (Burín, 2002) (Fernández, 1993), los que “(...) trabajan en el análisis (deconstrucción) de los consensos que históricamente han atribuido al malestar femenino causas demoníacas, biológicas, psíquicas, opacando las causas objetivas (su subordinación social) y confundiendo muchas veces efectos de tal subordinación (sintomatologías diversas, características subjetivas, etc.) con causas de su malestar" (Fernández, 1993, pp. 127-128).

Respecto a la Salud Mental, en términos generales la consideramos como un campo diverso y heterogéneo de posiciones, en el que hay capitales en juego, los que han sido reconocidos dentro del campo, y por consiguiente, disputados por los actores (profesionales, usuarios, entre muchos otros), que llevan a cabo diferentes prácticas ${ }^{4}$ ligadas al abordaje de la categoría de sufrimiento humano. Particularmente, a los efectos del proceso de investigación, adoptamos la propuesta de Burín (2002) de pensar el concepto de Salud Mental en clave femenina; retomando sus hipótesis más fuertes, la importancia de esta invitación refiere a revisar críticamente las prácticas que inciden sobre la Salud Mental de las mujeres y sobre sus modos de enfermar, la autora considera a la "(...) perspectiva sexista como productora de condiciones de vida enfermantes” (Burín, 1987, p. 37), resaltando la configuración del ser mujer, desde una perspectiva de género devaluado y desjerarquizado en nuestra cultura, salvo en la representación de la mujer-madre. Plantea la idea de Salud Mental de las mujeres como una situación de conflicto, cobrando gran valor la idea conceptual acerca de la construcción de género sexual femenino y su incidencia para con la Salud Mental de las mu-

4 Prácticas que siempre están en relación a su habitus-capital, en estado incorporado. 
jeres, como uno de los determinantes más importantes de la situación de conflicto; refiere así a que "tal situación conflictiva encuentra una de sus mejores formas de expresión en la constitución de la subjetividad femenina" (Burín, 1987, p. 30).

\section{Aspectos metodológicos}

Dadas las características del Objeto y lo propuesto para el estudio, la opción metodológica fue cualitativa, tipo de investigación caracterizada por subrayar la naturaleza socialmente construida de la realidad social, la íntima relación entre el investigador, aquello que estudia y los determinantes contextuales que condicionan la investigación. Desde dicha lógica es que se propuso, a través del relato de las mujeres, conocer en primera persona las particularidades que envisten las experiencias de las usuarias de Servicios de Salud Mental, donde se imbrican problemáticas concernientes a la Salud Mental, el género y la pobreza.

Trabajar con las narrativas de mujeres supuso un esfuerzo por producir conocimiento de la realidad social desde el punto de vista de quienes la producen, la viven y la significan. Siguiendo a Ana Lía Kornblit (2004), diremos que lo que se intenta es "comprender las significaciones otorgadas por ellos [los actores] a los hechos en estudio a partir de sus propias explicaciones [...], el propósito es reconstruir la lógica que anima sus puntos de vista" (Kornblit, 2007, p. 10).

Así pues, a partir de nuestro interés por conocer los significados que las mujeres otorgan a su experiencia, apostamos a la realización de un estudio con énfasis en lo biográfico narrativo, partiendo por comprender que:

La narrativa no sólo expresa importantes dimensiones de la experiencia vivida, sino que, más radicalmente, media la propia experiencia y configura la construcción social de la realidad. Además, un enfoque narrativo prioriza un yo dialógico, su naturaleza relacional y comunitaria, donde la subjetividad es una construcción social, intersubjetivamente conformada por el discurso co- 
municativo. El juego de subjetividades, en un proceso dialógico, se convierte en un modo privilegiado de construir conocimiento (Bolívar Botía, 2002, p. 4).

Al trabajar desde esta metodología se da un proceso por el cual el investigador interpreta el relato del sujeto entrevistado desde dos niveles (Lindón, 1999), su acervo de sentido común y sus intereses e inquietudes teóricas.

La investigación tuvo como unidades de estudio a Servicios Públicos de Salud Mental de las ciudades de Paraná (Entre Ríos) y Santa Fe, durante los años 2012 y 2013, donde se trabajó con mujeres usuarias de los Servicios de Salud Mental de dos hospitales públicos monovalentes de referencia zonal ${ }^{5}$, ubicados en las ciudades de Paraná (Entre Ríos) y Santa Fe (Santa Fe). El tipo de muestreo fue intencional, también conocido como selección basada en criterios; en algunos casos partiendo de vínculos previamente establecidos con usuarias durante procesos de años anteriores y ampliando, además, el universo hacia otros Servicios de Salud Mental, a los cuales se tuvo acceso por medio de informantes claves. Los criterios de selección fueron tres: a) Que se encuentren internadas o realicen un tratamiento ambulatorio en una institución de Salud Mental; b) Que tengan una larga trayectoria de asistencia en el campo de la Salud Mental (más de 5 años); c) Que tengan como institución de referencia el sistema público de Salud. A esto debe agregarse que la selección estuvo orientada por los equipos de salud intervinientes, en orden a poder acceder a relatos que sirvan a la investigación y con el fin también de preservar los tratamientos de cada una de las mujeres.

Se entrevistó a un total de 7 mujeres, todas ellas de más de 40 años, casadas y separadas de quienes fueron los padres de sus hijos. Solo una manifestó encontrarse en pareja al momento de la entrevista

5 Resulta significativo realizar esta aclaración, puesto que al trabajar en Hospitales de referencia zonal, nos encontramos con poblaciones que deben migrar y reconfigurar su cotidianidad a la hora de realizar tratamiento. 
y solo una no tuvo hijos. Sobre estos últimos, solo la mitad de las entrevistadas tienen trato cotidiano con ellos.

Para determinar el número de entrevistas se tuvo como horizonte el objetivo de lograr representatividad de los individuos seleccionados y, a su vez, captar la heterogeneidad de la población estudiada (Maxwell, 1996). Finalmente, en base a los criterios establecidos, se realizaron un total de 13 entrevistas en profundidad a 7 mujeres, de las cuales 3 se encontraban internadas y 4 realizando tratamiento de manera ambulatoria.

Con el propósito de cumplimentar con los objetivos de la investigación, se optó por la Entrevista Abierta (Souza Minayo, 1997) como técnica de recolección de datos principal. A su vez, se trabajó con registros realizados durante los años 2010 y 2011, con información relevada en entrevistas a los equipos profesionales, lectura de historias clínicas, entre otras. Los datos empíricos obtenidos fueron analizados apelando al método comparativo constante de Glaser y Strauss (2007) y recuperando las frases significativas (Badano y Díaz, 2010), como estrategia para no perder nunca el punto de vista de las mujeres entrevistadas.

\section{Hallazgos}

Si bien lo referido hasta el momento puede considerarse como eje vertebrador de la exposición, en tanto da cuenta de los articulados conceptuales que atravesaron todo el proceso de investigación, respecto de los hallazgos realizamos una necesaria selección a los fines de esta presentación particular. Al respecto, optamos por exponer aquello que en el ejercicio de la profesión se vuelve significativo, también en función de las nuevas lógicas que se encuentran orientadas por la Ley Nacional de Salud Mental No 26.657, así como se tornan aspectos representativos del sentido que tuvo el proceso de investigación, a partir de realizar un análisis impregnado de las Teorías de Género.

En este sentido, expondremos en primer lugar algunas reflexiones en torno a los modos de nombrar(se) presentes en los relatos, co- 
mo modo de condensación de relatos de otros y de producciones y reproducciones a partir de los mismos por parte de las mujeres. Seguidamente abordaremos la categoría locura desde una postura política, e intentaremos avanzar sobre su abordaje al interior de las familias.

Cabe aclarar que en tanto se citarán narrativas textuales de las entrevistadas, se ha optado por incluir nombres ficticios a los fines de preservar las identidades.

\section{Acerca de los modos de nombrar (se). ¿Qué me pasa a mí?, ¿estoy loca?}

En torno a la locura se dan complejos procesos de disputa de sentido, donde intervienen diversos actores y donde juega un rol crucial el diagnóstico médico en tanto producción de la verdad de la enfermedad (Foucault, 2007). A partir de allí se van generando múltiples formas de nombrar el padecimiento, muchas de las cuales tienen que ver con los diagnósticos. En este punto intentaremos reseñar las formas que interpretamos refieren a cómo es nombrado el padecimiento, atendiendo a la utilización de la categoría "locura" como apuesta política, buscando reconocer para poder deconstruir los significados compartidos sobre el sufrimiento psíquico que se hacen presentes en el lenguaje cotidiano. La idea de "locura" entonces debe ser pensada como categoría que condensa tantos sentidos y significados como relatos existan, estableciéndose diferencias, incluso, al interior del mismo relato de una entrevistada.

Analía, en su primera entrevista, se presentó diciendo: el diagnóstico es bipolaridad. Aparece, así, el diagnóstico como una carta de presentación, una característica que le brinda una identidad particular a Analía. Cuando nos habla de su diagnóstico refiere que le ha dado tranquilidad poder ponerle nombre a eso que le pasaba, después de haber consultado a muchos médicos, cada uno de los cuales la clasificó de manera distinta. Aun así, y si realizamos un recorrido por su relato, Analía habla con cierta disconformidad de su diagnóstico, expresando: el diagnóstico es bipolaridad pero en 
realidad debo decir era, dejando entrever que no sólo asume clasificaciones impuestas por otros (médicos en este caso), sino que ella misma se va construyendo, apropiándoselo por momentos y dejándolo de lado otras veces, no como algo acabado sino como algo en movimiento.

Mariana, en tanto, menciona que su internación tuvo lugar a partir de una crisis de nervios y Verónica, por su parte, cuando evoca los momentos de crisis o sus períodos de internación refiere: quedé internada por problemas de nervios, de presión o expresa haberse enfermado de depresión. En este caso particular, el reconocimiento de que "algo no andaba bien" es mencionado por la entrevistada, a partir de registrar ella misma cambios en su forma de ser, su cotidianidad, "yo estaba sentada y veía cómo la gente pasaba y pasaba, y decía: ¿qué me pasa a mí?, ¿estoy loca? Yo no hacía nada, estaba horas, $y$ horas y horas sentada" (Verónica 05/03/13). Si leemos detenidamente el relato de Verónica podemos interpretar que es a partir de una primera consulta médica que la entrevistada empieza a nombrar su padecimiento como depresión. A su vez, los términos biomédicos fueron utilizados recurrentemente al momento de la investigación para justificar, por ejemplo, posibles ausencias en su asistencia al Hospital en donde realiza tratamiento ambulatorio.

En este interjuego entre múltiples discursos, al que atendemos a partir de considerar los modos de significarse de las propias entrevistadas, consideramos pertinente tal como referimos al comienzo, problematizar la categoría locura en tanto categoría política, al asumir que en la misma se condensan aspectos, atributos y prejuicios, que son asignados a los sujetos que han vivido o viven situaciones de padecimiento subjetivo. Como menciona Robert Castel (1980):

[...] las nosografías se contentan con acuñar en un cierto número de subespecies esta percepción global y peyorativa. La locura, ese exceso que es una deficiencia: agitación, desmanes, arrebato, desmesura, desborde, impulsividad, imprevisibilidad, peligrosi- 
Narrativas de mujeres que transitan la experiencia de la "locura". Un recorrido por los itinerarios terapéuticos en Salud Mental / Benedetti y Kendziur

dad; estos rasgos están muy lejos de la apacible plenitud de una vida que haya integrado todas las normas disciplinarias configurando una segunda naturaleza. El etiquetamiento nosográfico no hace así más que formalizar los datos inmediatos de la conciencia social de la locura (Castel, R.; 1980: 125).

En la historia, se ha vinculado a los "locos" con la "animalidad", con lo "no humano", justificándose con ello formas de intervención represivas, a la vez que se acompañó el término con sentimientos, de "lastima" en tanto el sujeto vinculado a experiencias de este tipo contiene como característica primaria el haber perdido lo más valioso de lo humano, esto es, la "razón". Ocupa un lugar destacado en estos procesos la instauración del paradigma de "normalidad".

Retomando a Robert Castel (1980) respecto de los alienados, diremos que, hacia fines del S. XIX y principios del XX se conforma la idea de alienación como un "estigma pegado en la piel para toda la vida", lo que nos permite problematizar acerca del poder de los psiquiatras y el peso de sus diagnósticos, desde la perspectiva del control social ${ }^{6}$.

6 Mención aparte merece para esta investigación el par locura-mujer, para lo cual fueron tomados aportes de Mabel Burin (1987) en orden a poder desentrañar, reflexionar acerca de las particularidades que se ponen en juego y que tienen lugar hoy, pero que evocan los distintos procesos históricos y, en ello, los aspectos sociales, culturales, económicos y políticos por los cuales se ha transitado. Así, desde un abordaje en clave histórica, en épocas tempranas, ligada la vida a fuertes preceptos de la tradición judeo-cristiana y a una forma de producción religiosa, la idea del sujeto mujer en su vinculación con la salud mental atendió a dos roles opuestos, siempre a confirmación de la mirada de los hombres; por un lado, se encontraban aquellas mujeres vírgenes y, por otro, quienes eran dueñas de un carácter demoníaco, relacionado lo mismo con la sensualidad, lo irracional y -durante la Edad Media- con la disidencia política -llevando al extremo de la cacería de brujas-.

Desde una reconstrucción histórica del término vimos cómo, siempre a juicio de otros, las mujeres fueron tomadas y perseguidas cuando sus prácticas no eran coincidentes con los roles esperables de época, es decir, cuando no contaban con los atributos aprobados por el conjunto social. Ahora bien, ya a mediados del S. XVIII las concepciones sobre la salud mental fueron siendo reemplazadas por una idea ligada al binomio racionalidad/irracionalidad; allí la locura quedó excluida del mundo mercantil, de la moralidad y del trabajo.

De la misma forma, se produjeron transformaciones desde lo que fueron las casas medievales hasta la constitución de la familia burguesa, en donde lo que antes era considerado como labor productiva y reproductiva de las mujeres, fue perdiendo valor debido a que en los primeros años de la Revolución Industrial comenzó a ser 
Así, pudieron reconocerse en los relatos modos de hablar de sí (o nombrarse), que encuentran sus raíces en la ciencia moderna, donde es recurrente la idea de equilibrio, norma, normalidad, frecuencia, entre otras. Un ejemplo claro lo brindó Analía al manifestar no estar en un eje cien por ciento, lo cual interpretamos contiene una idea de estabilidad plausible de ser alcanzada, que se vislumbra en la relación con otros y en su organización cotidiana. La entrevistada manifestó tener una ansiedad de sanación completa porque hay episodios todavía, son leves, pero hay episodios en los que tiene el resabio de una enfermedad mental.

Los modos en que las mujeres se narran en la enfermedad nos aproximan a ver las múltiples formas en que esta experiencia "es representada y evocada, en la cual las ocurrencias de la misma poseen un orden coherente y significativo, donde actividades y eventos se describen por referencia al sentido que tienen para las personas involucradas" (Idoyaga Molina, 2007, p. 11).

En este sentido, lo sustancial reside en que existen tantas formas de nombrar y nombrarse como relatos obtenidos, y que en cada uno de éstos aparecen formas diversas que se relacionan con los momentos narrados, los aspectos que las mujeres entrevistadas resaltan y los actores presentes en cada uno. Así, por ejemplo, Ana, al igual que Verónica, describe como síntoma el reconocimiento de algo que, según interpretamos, escapaba a su cotidianidad: “desconocía a mis parientes", lo cual la lleva a una primera internación; ahora bien, al momento de la entrevista, ella nombra su malestar a partir de la intervención de otros actores que desde el plano jurídico le realizan un juicio por insanía mental.

valorada como verdadero trabajo la producción extra doméstica únicamente. Se produjo, entonces, un estrechamiento del escenario doméstico y, junto con ello, un achicamiento del entorno del trabajo de las mujeres, sobre todo, en lo referido a la pérdida de perspectiva, en tanto las acciones tales como la crianza de los hijos, las labores domésticas, etc., pasaron a considerarse como "tareas naturales" acompañadas de una fuerte impronta moral. Es allí donde se conforma lo mencionado como "ideal maternal", lo que, como veremos en apartados siguientes, permea en algunas vivencias expresadas por las usuarias al vincular su padecimiento con experiencias ligadas a modos de relación familiar. 
Quisiéramos remarcar en este punto que la manera en que respondió a nuestras interrogantes respecto de la internación ofreció pistas para pensar que Ana no se nombra a sí misma como insana, sino que ésta es una forma de nombrar de otros que ella reproduce en tanto respuesta que justifica su permanencia en la institución.

Continuando, podemos ver cómo en los discursos aparecen categorías ligadas a la enfermedad, como enfermo mental, paciente psiquiátrico, etc., pero siempre haciendo referencia a un malestar y a una búsqueda constante por calmar ese sufrimiento,

"Es muy difícil la problemática del paciente psiquiátrico, es sumamente difícil, porque el paciente psiquiátrico no sabe cómo se va a sentir ese día, es algo que es una sorpresa, o sea no sabe si va a estar depresivo, si va a estar efusivo, si va a estar desganado, con ira, con bronca, no se sabe, es una caja de sorpresas el enfermo psiquiátrico" (Analía 24/04/13).

Resulta muy interesante poder ver cómo las formas de hablar de sí mismas presentaron similitudes y singularidades en función del momento transitado, narrado por las mujeres. Así, el término locura apareció mencionado en el relato de Verónica al describirse su situación previa a la consulta médica y también se referenció en la voz de la entrevistada al narrarnos las experiencias que ella vincula a la producción de su malestar:

“(...) entonces él, con todo el drama que yo tenía con mi mamá, que estaba enferma de cáncer, él se iba a Concordia, me dejaba con los chicos, se iba a estar con ella y me quedaba sola con los tres chicos, y me quedaba enferma y loca dentro de casa" (Gloria 03/06/13)

“(...) O sea, es como que... siempre tenía cosas para decir de mí, siempre tenía quejas en relación a cómo me criaban mi papá y mi mamá: "que yo era una malcriada, una mal enseñada, que no tenía disciplina, que lo que a mí se me antojaba yo lo tenía, mientras que los hijos de ella no", todas situaciones que 
terminan en envidia, en resentimiento... y una termina loca y hasta el día de hoy me hace mal, no quiero ver a nadie porque a mí me hace mal..." (Gloria 03/06/13)

Las distintas formas en que las mujeres hablan de sí respecto a su malestar nos permitió poder reflexionar sobre los significados que construyen y cómo a partir de allí se presentan; ver cómo hacen jugar en sus relatos diferentes actores, espacios y tiempos, percepciones que, en definitiva, construyen un relato singular, propio, único. Por otro lado, comprendemos que existe un interjuego en los sentidos que imprimen las mujeres en sus relatos, donde por momentos hablan de sus experiencias particulares y se distinguen del conjunto, pero en algunos casos se describen en función de quienes comparten, por ejemplo, sus espacios y actividades dentro del Hospital.

"Lo que pasa es que acá hay gente que está un poco mejor que la gente que está en el Hospital internada, por ahí vienen algunos que están mal también pero yo estoy acostumbrada, estoy acostumbrada a toda esa gente de acá, hace años que vengo acá... Ya conozco a todos...” (Verónica 05/03/13).

"Porque ustedes se habrán dado cuenta que los enfermos no es que estamos -y me incluyo porque yo todavía no estoy dada de alta- todos dados vuelta... Hay personas con las cuales sí no se puede hablar, no se puede tener un vínculo, pero hay muchas otras que están en proceso y sí se puede tener vínculo, y el afecto y la compañía y el acordarse de ellos es muy importante..." (Analía 16/07/13).

Gloria, por su parte, se incluye dentro de un colectivo de personas que venimos al Hospital, que tienen trastornos de conducta o trastornos mentales, y algunas otras que somos demasiado sensibles y no sabemos defendernos del entorno (Gloria 30/04/13). Esta usuaria, además, se incluye dentro del colectivo "discapacitados", en tanto categoría a la que se incorporan determinadas personas por medio del reconocimiento del Estado, fundamentalmente a partir del vínculo con determinadas políticas. Al respecto, consi- 
deramos que "las políticas están profundamente implicadas en la manera como nos construimos como individuos y como sujetos. Para decirlo de otro modo, uno de los aspectos más importantes de la formulación de las políticas públicas es la forma en que éstas construyen nuevas categorías de subjetividad y nuevos tipos de sujetos políticos; al decir de Shore (2010), las políticas construyen activamente identidades. No obstante este señalamiento al cual atendimos para realizar la investigación, observamos que, en función de la dialéctica entre los modos en que las mujeres se reconocen a sí mismas y los modos en que son reconocidas a lo largo de sus itinerarios terapéuticos, pudimos atender a configuraciones subjetivas que interpelan -a la vez que definen desafíos a las fronteras entre el campo de la discapacidad y locura, esto es, que discuten los modos de categorización que se imparten desde el Estado, proponiendo también otros recorridos.

Como dato interesante desde la misma lógica, si bien en los relatos existen variadas menciones que hacen pensar en la existencia de un malestar de género por verse obstaculizada la posibilidad de acompañar, asistir y cuidar a los hijos como "deber ser", también se perciben posturas que, aunque son escasas, permiten pensar que el rol materno atribuido no las define aunque se encuentre presente.

Nos interesó, entonces, poder traer a colación algunas de las múltiples formas en que las mujeres hablan de sí, se definen en relación a su padecimiento y con esto reflexionar acerca de cómo se apropian o resisten ante las clasificaciones impuestas desde afuera. El hecho de que sean capaces de narrar sus propias historias nos da pistas más que claras de que no estamos ante vidas narradas solamente por otros (médicos, diagnósticos, políticas sociales, etc.), sino que estamos ante un proceso activo de construcción y disputa de significados en donde son ellas también las que reflexionan sobre sus experiencias en un contexto sobre el que también disputan sentido. Un ejemplo claro lo brinda Gloria, quien habla de sí como delirante en un entorno violento; menciona a su malestar en función de un entorno trágico, de una sociedad que para ella es 
horrible, espantosa y a la cual critica, pero que al final "uno se queda como se quedaba el Quijote peleando con los molinos de viento" (Gloria 30/04/13).

\section{La "locura"... un asunto de familia}

En tanto partimos de entender a la Salud Mental como Campo, se vuelve necesaria la reflexión acerca de los actores que inter juegan constantemente allí, así como también debemos poder comprender la historicidad de su construcción, viéndose implicado con esto, diferentes perspectivas, algunas coexistentes y otras que dan y dieron lugar a modificaciones. En este sentido, a partir del análisis de los relatos, consideramos que la familia emerge como actor protagónico en el tratamiento de la "locura", donde adquiere una cierta particularidad referida a los lugares desde los cuales la misma se ha relacionado con el padecimiento.

Tradicionalmente la familia ha tenido un lugar privilegiado en torno a las elecciones y decisiones que toman los sujetos en la búsqueda de solución de los problemas relacionados con la salud, es decir, cumplen un papel importante en los itinerarios terapéuticos, ya que todos somos seres sociales que, por tanto, optamos casi siempre habiendo previamente puesto en discusión o considerado las opciones que existen para emprender el camino de sanación.

En el tratamiento de la "locura" advertimos una particularidad que refiere a que los tránsitos de los pacientes muchas veces no son iniciados por ellos mismos, sino sugeridos o pedidos por las familias, sujetos allegados, órdenes judiciales, etc. Si miramos este proceso a partir de lo que Ervin Goffman (1998) denominó la carrera moral del enfermo mental, veremos que en esta primera instancia, denominada etapa de pre-paciente [...] un grupo relativamente reducido se interna de buen grado (...) (Goffman, 1998, p. 136).

En el caso de las mujeres entrevistadas, los relatos respecto a las internaciones y a los sujetos implicados en tal situación, fluctúan entre quienes intentan mostrar cómo la internación fue inducida por la situación que estaban viviendo, y quienes, en forma explíci- 
ta, expresan disconformidad con la decisión, pues habría pesado más la voz de sus familiares que las suyas.- ¿Vos querías venirte? - "No, no, no... yo había alistado dos o tres bolsas de ropa [...] no dormí yo esa noche, esa madrugada no dormí [...] al día siguiente, después entré acá, me enchufaron..." (Dora 12/06/13).

También interpretamos que el hecho de encontrarse en una situación de padecimiento muchas veces pone en duda la voz de las mujeres, quienes se encuentran ubicadas en un lugar de inferioridad a la hora de tomar decisiones de incumbencia familiar, como en el caso de Laura, que relata: "[...]Estuve internada el año pasado y mi hermana vendió las herramientas de la gomería y no me avisó nada, y a mí me dieron por 6 meses la casa y ella la vendió y me van a llevar a Tribunales de vuelta para ver cómo seguimos los trámites (Laura. 13/09/12). O Verónica, quien al hablar de su situación familiar actual nos dice (respecto de las cosas que le molestan): “-Sí, lo hablo, pero por ahí me tratan medio mal, entonces no hablo nada” (Verónica 05/03/13).

Sobre esta última y otra de las entrevistadas -Gloria-, merece la pena reseñar que su situación familiar estuvo signada por la violencia, particularmente la violencia contra la mujer (Femenías, 2013), por lo cual este acallamiento que se menciona en referencia a las voces de las usuarias sobre su situación, puede pensarse reforzado por procesos de violencia que tienen su fundamento en desigualdades entre los géneros; podríamos pensar que quizás esto se haga extensivo a la totalidad de las situaciones abordadas, pero como no contamos con datos suficientes, preferimos plantearlo como interrogante.

Ahora bien, partiendo de comprender el rol fundamental que ha desempeñado la familia en la historia del tratamiento de la locu$\mathrm{ra}^{7}$-el cual ha mutado y se ha transformado-, desde los relatos de

7 De forma sintética, y realizando una lectura histórica de la construcción del Campo de la Salud Mental, diremos que durante el Antiguo Régimen la legislación de la locura se concebía como un asunto de familia, con intervenciones desde lo jurídico y administrativo en caso de ser necesario; a ello se agrega con la instauración de un 
las entrevistadas comprendemos que en sus experiencias narradas es posible reconocer vestigios de diferentes concepciones respecto de la salud y enfermedad mental y su tratamiento. Así, Verónica, por su parte, nos permitió reconocer una relación - ¿o sentimiento?- de cierto "paternaje" por parte de su médico a la vez que expresó contar con un escaso apoyo de sus familiares durante su estar en el Hospital: "Yo tenía un médico muy bueno cuando estaba en la sala, muy bueno. Conmigo, por lo menos conmigo era muy bueno. Era un hombre grande, me preguntaba si estaba bien, todo lo que un médico te tiene que preguntar [...] Se preocupaba por mí, aparte me decía: -Cuando quieras internarte vení y te interno... [...] Y aparte él me ayudó con el tema de la pensión (...)" (Verónica 03/06/13).

Respecto del punto que nos interesa aquí y pensándolo en términos históricos de tratamiento de la "Locura", vemos cómo la figura del médico reemplaza paulatinamente a la familia como aquella institución responsable por sus "locos", sin embargo, lo mismo no implica desconocer el papel fundamental que cumple esta última.

En los relatos de las entrevistadas, si bien pueden aparecer difusos los personajes que, por ejemplo, encaminaron hace 8 años a Dora al Hospital en el que se encuentra internada, o puede que no se manifieste de forma explícita quiénes fueron los que llevaron a Mariana al lugar, entre otros casos, podemos interpretar a partir de las entrevistas, cómo el reconocimiento de que "algo no andaba bien" fue realizado por familiares.

Por otro lado, si bien se promociona actualmente a la familia como actor importante dentro del proceso salud/enfermedad/cuidados de los pacientes, en momentos de internación, externación y tratamiento ambulatorio, existe mayormente lo que se interpreta como desprendimiento frente a las usuarias al momento en que son trasladadas a la internación. Este planteo puede quizás vis-

nuevo orden, el poder médico, dando lugar a una serie de modificaciones, con lo cual se asiste al triunfo de la medicalización, donde los médicos comienzan a cumplir un papel cada vez más importante. 
lumbrarse en los relatos de Laura y Verónica: "Sí, me quería ir, y yo la llamaba a mi hermana y no venía, y no venía” (Laura 13/09/12), "No quieren venir acá conmigo. Yo tengo dos hijos... Una mujer y un varón. Nunca me acompañan” (Verónica 18/09/12).

Pareciera existir en casi todos los casos una imposibilidad o dificultad por parte de las familias para poder acompañar a las mujeres en actividades del Hospital cuando éstas lo solicitan, como que si lo que pasa allí correspondiera únicamente a vínculos entre el personal de la Institución y los usuarios.

Ahora bien, a partir de lo anterior, vale resaltar que según Foucault (2007), el Asilo rompe con la familia, ocurriendo, con la Ley 1838, una "desposesión de los derechos de familia con respecto al loco" (Foucault; 2007, p. 114). De la misma forma, hasta el S. XX el saber técnico sostuvo que de ninguna manera un alienado podría ser curado en su seno familiar sino que, y por su contrario, prevaleció con el aislamiento el principio de la disociación que justificaba que el loco nunca debía pensar en su enfermedad para poder sanarse, es decir, se situaba al loco en un mundo totalmente ajeno.

Conjeturamos que la concepción expuesta puede estar manifestándose en la actualidad en tanto vestigio de las luchas que se han desarrollado entre los actores del Campo. Vale la pena aclarar que cuando nos preguntamos sobre el lugar de la familia evitamos formular juicios, para buscar dar cuenta de la relación dilemática, privilegiada, difícil y problemática en palabras de Foucault (2007), que el asilo ha mantenido con las familias desde el mismo momento de su constitución. En el caso de Verónica también nos expresa: "Yo vivía con el padre. Pero... después de la internación, esa primera internación que tuve yo, él no quería que fuera más... a estar con los chicos" (Verónica 18/09/12); como si directamente se la expulsara de su seno familiar debido a su situación.

Por otro lado, al realizar un análisis de los vínculos que se suscitan entre las mujeres y sus familiares no podemos perder de vista que los lugares en los cuales se construyeron los asilos y las distancias desde estos hacia cada hogar pueden actuar también como im- 
pedimento para el sostenimiento y/o reconstrucción de los lazos familiares.

Esto se interpreta sobre todo en lo narrado por dos de las mujeres que se encuentran internadas, una de las cuales refiere: "[...]Soy de Las Toscas...Provincia de Santa Fe. Son 7 horas de viaje..." (Dora 12/07/13); por lo cual podemos interpretar que las distancias dificultan las visitas de sus familiares y de ella a su hogar: $-\dot{i}$ Hace mucho que no vas para allá?...” Y mirá, más o menos hará, esperá que piense: octubre, noviembre, diciembre, enero, febrero, marzo, abril, mayo... 8 meses" (Dora 12/07/13). En contraposición, es importante destacar que Analía cuando recuerda una experiencia de crisis lejos de su hogar nos cuenta: "Allá en Buenos Aires me acompañaron mis hijos; mis hijos fundamentalmente, y mi madre. Mi madre me acompañó mucho en la internación" (Analía 19/06/13).

Respecto de lo narrado sobre la vinculación de la familia con el tratamiento en el ámbito del hogar, las experiencias son variadas, desde sentimientos de soledad (Laura 13/09/12) hasta de cuidados y apoyo con la medicación (Verónica 05/03/2013).

Así, si bien existen particularidades respecto de los modos de relación que se construyen entre las familias y sus "locos", en tanto asistimos a experiencias y subjetividades singulares, entendemos que también pueden ser reconocidas algunas cuestiones que se comparten, como por ejemplo, la tensión permanente entre la demanda de los pacientes de atención de sus familiares y una repuesta por parte de estos que, como vimos, muchas veces no cumplen las expectativas de las entrevistadas. Comprendemos que algunas formas de trato y de relación al interior de las familias, reflejan algunas concepciones históricas que se expresan como representaciones y acciones concretas que dificultan la integración familiar y dan cuenta de las relaciones dilemáticas que se tejen en torno al tratamiento de la locura. Con esto hacemos referencia a la existencia de formas de relación diversas entre las que pudimos reconocer tratos violentos hacia las mujeres vinculados al padecimiento, acallamiento de la posibilidad de expresión de las usuarias, inten- 
tos de separación de las entrevistadas de sus hijos fundamentado ello en la presencia de enfermedad, dificultad en el acercamiento de familiares a los tratamientos una vez que se logra la internación, así como también a situaciones en donde los familiares se apropian de bienes - para su uso o venta- desde una lógica de acción que da cuenta de representaciones sobre los sujetos con padecimiento, resultando en una especie de anulación de los mismos.

\section{De presencias y ausencias}

Quisiéramos detenernos ahora y reflexionar acerca de los movimientos (modificaciones y continuidades) que se produjeron en las familias a partir de las experiencias vividas por las mujeres en sus itinerarios terapéuticos. Nos referimos a las huellas y cambios que se dan a partir de la situación de malestar, las que son vividas por las mujeres como experiencias de quiebre.

Para este punto, retomamos a Natalia Castelnuovo (2006), quien, realizando una recopilación de perspectivas que en la Antropología han definido a la familia [y, en esto, las implicancias de tales teorías para el total de las Ciencias Sociales] expresa, que "El desafío [...] consiste entonces en dejar de pensar en la "familia ideal" como un mundo que hemos perdido, y reconocer que si las familias se hallan en constante transformación, es anacrónico hablar de La Familia y, en cambio, como apuntó Bestard, sólo es posible hablar de formas familiares" (Castelnuovo; 2006:56). En esta línea, define que la familia es una organización social conformada por tres dimensiones en constante transformación: la sexualidad, la procreación y la convivencia.

En primer lugar, sobre la convivencia, vale retomar lo trabajado en el punto anterior acerca de la ruptura que la institución asilar produce en relación con la familia. También, en un mismo sentido existen expresiones que refieren a situaciones de quiebres previos en lo familiar que dan lugar a la finalización de la convivencia; tal como expresó Gloria. 
(...) Era una situación que ya no se podía sostener más, entonces yo me junté con los tres chicos y me fui de la casa, hice abandono del hogar (...) (Gloria 03/06/13).

Cabe aclarar que no pretendemos situar la ruptura de la convivencia como un hecho estático, de una vez y para siempre, sino que en varias situaciones los itinerarios de las mujeres que reflejan sucesivas internaciones nos permiten pensar en procesos diferentes en donde se vislumbran posibles nuevos formatos de estar en familia.

En segundo lugar, en razón de las dos primeras dimensiones, si bien desde una perspectiva de género conocemos $y$, a su vez, somos críticas del lugar en que se sitúa a las mujeres en el todo social y dentro de éste, en la familia ${ }^{8}$, llama la atención lo relatado por las entrevistadas y no podemos desconocer que todas aquellas cuestiones que se les demandan culturalmente, pueden producir malestar cuando por su padecimiento, se encuentran "ausentes". Este "ausentarse" refiere en principio a la no convivencia pero, en función de lo recabado, indica fundamentalmente no estar presente en las familias para el cuidado, el no contar por momentos con ingresos con los cuales aportar para alimento de los hijos, no acompañarlos en sus crecimientos, entre otros.

En este sentido, como plantea Mabel Burín (1987), "la Salud Mental de la Mujer tiene su propia especificidad", lo que determina que existen prácticas sociales que inciden sobre la salud mental de las mujeres y sobre sus modos de enfermar y sanar. Así, la autora propone incluir en la problemática de la Salud Mental, la perspectiva sexista como productora de condiciones de vida enfermantes (Burín, 1987, p. 37), en tanto la mujer es un género devaluado y desjerarquizado en nuestra cultura, salvo en la representación de la mujer-madre.

Teniendo en cuenta lo mencionado es que nos cuestionamos acerca de lo que ocurre en las familias cuando quien ocupaba el rol de

8 Es decir, llamadas a cumplir su rol maternal, de cuidado y atención de los quehaceres del hogar. 
madre, se encuentra, por lo menos momentáneamente, impedida de seguir cumpliendo con las actividades demandadas. Si bien no pretendemos dar una respuesta acabada intentaremos exponer y reflexionar acerca de una de las situaciones de las mujeres entrevistadas. En principio, escogemos traer a esta publicación la situación de Verónica, ya que interpretamos más claramente, en el relato de sus experiencias, la ruptura que produce el Hospital, fundamentalmente la situación de internación, en la vida familiar.

- ¿Y tu hija terminó la escuela? - "No, fue hasta segundo año". - ¿Y vos sabés por que no fueron más? - "Porque yo estaba internada" (Verónica 03/06/13).

En este caso, en el que la entrevistada relata su vivencia referida a sus primeras internaciones, pareciera que relaciona su "no estar" en el hogar con algo que puede interpretarse como una ruptura en la cotidianidad de sus hijos y que quizás implique un malestar en estos. De la misma forma, cuando habla de ellos en la actualidad menciona que "[...] hablan. Hablan y se pelean así por el tema mío, viste”; ligándolo explícitamente a una situación de conflicto.

A su vez, podríamos interpretar que se vivencian sentimientos de culpa por no haber podido estar con sus hijos: ¿Y con quien se quedaban los chicos cuando estabas internada? -"Con el padre. Ellos tendrían 12 o 13 años, se quedaron un tiempo solitos también, pobrecitos...”. Además de dejar entrever que pone en cuestión la capacidad de cuidado del padre hacia éstos: ¿Y los chicos vivían con él nomás o con alguien más? "-Con él nada más, pero él los dejaba solos..." (Verónica 18/09/12).

En los encuentros que mantuvimos, podemos interpretar que Verónica intenta reafirmar la importancia de su rol al interior de su familia, ya sea antes de la internación, en su labor como ama de casa y en el tiempo de internación. "Yo tenía un plan acá, que limpiaba todo acá arriba, yo, en ese tiempo. Estuve dos años y entonces con esa plata yo los ayudaba a ellos. Iba y les llevaba la plata a ellos. Mientras anduve bien, mientras podía" (Verónica 03/06/13). 
Sobre esta situación creemos que, si bien reconoce que existieron modificaciones suscitadas en su familia a partir de su padecimiento, ella no se describe como ausente sino que intenta en parte remarcar los aspectos a través de los cuales pudo mantenerse en vinculación cercana con sus hijos.

Para sintetizar, si bien cada mujer que ha vivido y vive la experiencia de la maternidad relata cuestiones muy diversas, creemos importante destacar que cuando no son ellas, las entrevistadas, quienes cumplen el rol de mujer-madre, por lo general aparece otra figura femenina que se expresa en términos de reemplazo ("su otra mama" tal como formulara Gloria) o colaboración ("Entonces vos la criaste sola... Sí... Bah... la crió mi mamá”, al decir de Mariana). Cuando se menciona la figura paterna en términos de crianza, la misma es vinculada a situaciones de tensión como peleas familiares y tratos violentos o, como vimos en el caso de Verónica, directamente se ponen en cuestión las capacidades de cuidado.

\section{Acompañamiento y cuidados en los itinerarios terapéuticos: ¿asunto de mujeres?}

Como ya hemos expresado, los tránsitos de los pacientes se encuentran sujetos en gran medida a las opiniones, sugerencias o, en el caso particular de Salud Mental, a decisiones de otros. Así es que, durante el trabajo de campo, surgieron menciones e indagamos acerca de los actores que las mujeres reconocen como importantes en su proceso de salud-enfermedad.

Los hallazgos apuntan a poder reconocer que existen fuertes componentes genéricos en las acciones de acompañamiento y cuidado, ya sea respecto de los sujetos que las mujeres mencionan como colaboradores en su situación, como en las demandas que las mismas realizan hacia sus familiares.

Para este punto tomaremos el concepto de "Cuidados Familiares", el cual, según expone Rosario Aguirre (2009), ha resultado de importancia para mostrar el papel de "las familias como mecanismo de protección social que debe combinarse con las acciones del 
mercado y del Estado" (11). Se le define como la acción de cuidar un niño o una persona adulta o anciana dependiente para el desarrollo y el bienestar de su vida cotidiana. Si bien implica un trabajo material también se reconoce el aspecto afectivo y emocional que conlleva. La actividad puede ser realizada en la familia o puede ser delegada a otras personas ajenas a ella y puede ser remunerada o no.

Así, respecto de los relatos de las entrevistadas, si bien Verónica repite en sus encuentros que sus hijos no quieren acompañarla al Hospital, reconoce que en su casa "la ayudan". Sobre ello, en términos generales habla de una ayuda en plural pero luego especifica que es su hija quien colabora con ella, a diferencia del varón que parece no atender a la necesidad de ayuda de su madre.

"Sí, me ayudan, porque por ahí yo estoy loca y me da ganas de tomar todo junto, cuando me empiezan a pasar cosas así, ¿viste? Me empastillo... -Y ahí aparecen ellos para cuidarte...-. Sí. Yo antes le daba las pastillas a mi hija para que me las tenga, ya las conoce. Ella también me ha comprado, cuando se me perdían o me tomaba a veces de más. Y ahora no hago más eso porque si no me voy a ir al tacho..." (Verónica 05/03/13). De la misma forma, su hija la acompaña a cobrar la Pensión Nacional "A ella, a mi hija le pedí que me acompañara. Entonces me pide $\$ 100$ ella. Por el mandado..." [sonríe] (Verónica 18/09/12).

Creemos que resulta significativo lo anteriormente citado, en tanto este "pago" de madre a hija tiene que ver con un reconocimiento de ambas de la práctica de ayuda/acompañamiento que esta última realiza.

A su vez, vale la aclaración sobre el caso particular de Verónica quien pide ayuda referida a su tratamiento o a sus actividades sólo a su hija. En cambio, los pedidos hacia su hijo se corresponden con la realización de aportes a la economía del hogar. En el caso de Laura, quien solo cuenta con un hijo varón, al mismo se le solicita mayormente aportes monetarios o de recursos en general (alimentos), y respecto a los pedidos de asistencia en el tratamien- 
to, por medicación o acompañamiento, así como en actividades cotidianas, las ayudas se extienden a mujeres cercanas (hermana, madrastra del hijo).

Analía por su parte, resalta la presencia de su madre durante su primera internación: "Allá en Buenos Aires me acompañaron mis hijos; mis hijos fundamentalmente y mi madre. Mi madre me acompañó mucho en la internación”; también enuncia el acompañamiento por parte de sus hijos allí y es la única de las entrevistadas que otorga un papel importante y positivo en su vida a sus parejas, resaltando la ayuda que ha recibido en determinados momentos de su proceso de salud-enfermedad.

En el caso de Verónica aparece nuevamente una vecina, y, si bien ella se encontraba conviviendo con su ex esposo, es esta última quien advierte, da aviso de su situación, la acompaña y ayuda durante su primera crisis. "Entonces mi vecina, por hacerme un bien a mí, ella me dijo que era para un bien mío, porque yo andaba muy acelerada, muy nerviosa. Entonces llamó al juzgado y me acompañó y todo, fue y me buscó en su auto y me trajo" (Verónica 05/03/13).

La fuerte presencia de mujeres en situaciones claves expuestas por las entrevistadas, tiene que ver con que el cuidado en la sociedad "es una función adscrita a las mujeres como parte del rol de género; el cuidado de la salud de las personas que lo necesitan es cosa de mujeres" (García-Calvente, 2004, pp. 2-3).

Si bien el concepto de Cuidado Informal según la autora citada, se aleja de las experiencias narradas por las mujeres en tanto el mismo refiere puntualmente a un "asunto de familia", resultaba importante a nuestro juicio poder retomar lo expuesto. Esto tiene que ver con no dejar pasar el hecho de que en los casos en los que las entrevistadas no poseen un familiar directo que colabore en determinadas situaciones, también fueron y son mujeres las que brindaron su ayuda.

Respecto al trabajo doméstico y tareas de cuidado (Aguirre, 2009) en las entrevistas se visualizan como prácticas en que son las mu- 
jeres quienes mayormente otorgan sus servicios, lo cual tiene que ver con la construcción social que existe de "ser para otros", y donde sus actividades y deseos se relacionan intrínsecamente con un ideal maternal, de crianza y cuidado que se piensa y reproduce como natural, llevando en muchos casos a una falta de reconocimiento social.

\section{Conclusiones}

A modo de cierre y con vistas a abrir nuevos interrogantes que permitan profundizar en lo específico de los procesos de saludenfermedad-atención y cuidados de mujeres, existen algunas ideas que creemos pertinente retomar.

A partir del proceso de investigación nos encontramos con relatos de itinerarios terapéuticos de mujeres, donde el ser mujeres, pobres y "locas" las sitúa en circuitos complejos en que se refuerzan los estigmas y estereotipos propios de estas categorías, como procesos que devienen del juego articulado entre las mismas. Así, si bien no ha sido abordada con profundidad la noción de pobreza, en los relatos de, por lo menos, la mitad de las mujeres, éstas plantean en reiteradas oportunidades, las limitaciones económicas -previas y al momento en que registran el inicio de su malestar-como un elemento obstaculizador de sus desenvolvimientos cotidianos, siendo que al ingresar en el Sistema de Salud se abren a los beneficios de políticas específicas del sector, que al tiempo que brindan otro tipo de posibilidad económica, también las encierra dentro de la categoría "discapacidad"; allí se limita, en un mismo movimiento, la posibilidad de obtener otros trabajos pero, a la vez, se constituye en una opción monetaria desde la cual aportar a sus familias. Al mismo tiempo, aquellas situaciones de cobro con contraprestación, las vincula con tareas históricamente adjudicadas a lo femenino (limpieza, servicio, etc.); cabría aquí la pregunta para profundizar en nuevas líneas de investigación sobre lo específico de la interacción de estas políticas en la población de varones usuarios. No obstante lo dicho, también es loable reconocer que en la reconstruc- 
ción de los itinerarios terapéuticos pudimos visualizar posibilidades que tienen que ver con la sanación, con la sobrevivencia y el reconocimiento.

Respecto de los itinerarios terapéuticos vimos que son sinuosos, no son lineales, son particulares y significados de modos distintos por cada una de las mujeres; ellas nos hablan de sufrimientos, pero también, de luchas por calmar ese sufrimiento, el que no es más que y tanto como las diversas maneras en que significan su padecimiento subjetivo. Cada una logró establecer en sus relatos, las formas distintas de autopercepción de aquello que "no andaba bien" y significan búsquedas propias que alivian su acontecer ligado a tratamientos de atención del Sistema de Salud. Los tránsitos, en este sentido, fueron desde la búsqueda de ocupaciones al interior del Hospital al momento de las internaciones y aun durante el ambulatorio, así como actividades externas que luego terminan compartiendo en los espacios de Salud; tal es el caso de Gloria, que aporta desde sus conocimientos en las artes plásticas o Analía, quien se volcó hacia un trabajo sobre su espiritualidad.

$\mathrm{Al}$ conocer los Itinerarios pudimos visibilizar las tensiones que se ponen en juego allí: entre diagnósticos, políticas que parecen categorizar, clasificar e historias particulares donde, si bien hay mucho de resignación, también aparece algo que se pone en movimiento, se cuestiona, a partir de los múltiples tránsitos de las mujeres entrevistadas que dan lugar a una puesta en juego de sus capitales, logrando dar un sentido propio al tránsito por el sistema de salud, a las categorías que constituyen procesos de encerramiento y estigma y hasta la lucha por la puesta en valor de sus voces en sus vínculos cercanos. Aquí, algunas de las condiciones previas que en los relatos aparecen ligadas de forma estrecha a la enfermedad, se vinculan con experiencias de depresión y/o enojo frente a conductas esperables y estereotipos:

“(...) entonces yo hasta el día de hoy tengo las fotos que reflejan cómo mi mamá me veía, o cómo era su expresión de deseo de que fuera yo también..." (Gloria 03/06/13). 
"Trabajé acá en servicio doméstico también, y trabajaba él también. Antes que nacieran los chicos, cuando nacieron ya se me puso difícil la situación. E: Ahí fue cuando empezaste a quedarte más en tu casa... V: Ahí fue cuando me quedé en mi casa y ahí me enfermé, me enfermé de depresión (...) (Verónica $05 / 03 / 13$ ).

En sus relatos, las mujeres fueron evocando diversos escenarios, actores, consideraciones y valoraciones respecto de los tratamientos que emprendieron y de los espacios por los que han transitado y los que transitan aún hoy. Por fuera de las singularidades, en estas historias existe una cualidad que transversaliza toda la muestra. Esta se relaciona con que todas las mujeres atendieron a procesos de adjudicaciones de otros, lo que entendemos fue mediado por sus propias oposiciones y representaciones. Así, en esta concepción de los sujetos como construidos por otros, cabe significar una posición activa de parte de las mujeres entrevistadas. Como sostuvimos desde el comienzo, cada práctica en salud construye un sujeto determinado al cual le destina un tipo de intervención particular. En esta construcción se condensan y mezclan diversos paradigmas, culturas y modos de percepción del otro que dotan a éste de un conjunto de atributos que pueden potenciarlo o estigmatizarlo, lo que influye en los diversos procesos de configuración de subjetividades particulares. Cabría entonces aquí plantear como interrogante a profundizar en futuras líneas de investigación, si el padecer de algunas de las mujeres no tuvo que ver con un enfermar como resistencia ante roles adjudicados culturalmente, es decir, si en un mismo proceso aquello que las pone en un registro de padecimiento puede ser considerado, al mismo tiempo, una herramienta reguladora de preservación de salud.

Al hablar de subjetividades construidas no podemos, entonces, perder de vista la particularidad que le imprime el hecho de que las que narren sean mujeres, en una cultura que decide y define cómo deben ser. Sobre esto, nos propusimos dar cuenta de los relatos de mujeres que transitan la experiencia de la "locura", a partir de considerar las particularidades que representan las subjeti- 
vidades femeninas, entendiendo que en el proceso se entretejen elementos, ideas, supuestos y prácticas de carácter socio-histórico que se internalizan, así como también aspectos de la experiencia vivida por cada mujer en su tránsito por la "locura", donde se construyen itinerarios terapéuticos singulares.

Las distintas formas en que las mujeres hablan de sí respecto a su malestar, nos permitió poder reflexionar sobre los significados que ellas mismas construyen, y cómo, a partir de allí, se presentan. A lo largo de este camino recorrido nos hemos encontrado con diversas historias, historias intensas, que tienen por protagonistas a mujeres que sufren, caen, viven momentos difíciles y sin embargo, resisten, luchan, sobreviven. Mujeres que hablan de sí y de su historia rememorando tanto momentos difíciles como experiencias gratificantes, épocas felices, tiempos en los que acompañaron y se sintieron acompañadas, períodos en que se sintieron solas y decidieron salir adelante, sorteando situaciones de discriminación, estigmatización y exclusión.

\section{Bibliografía}

Aguirre, R. (2009). Las bases invisibles del bienestar social. El trabajo no remunerado en Uruguay. Uruguay: Doble Clic Editoras.

Argentina. Ley No 26.657. «Ley Nacional de Salud Mental» (2010). Boletín Oficial, No 32041.

Aquín, N. (2003). Ensayos sobre ciudadanía: Reflexiones desde el Trabajo Social. Buenos Aires: Espacio Editorial.

Badano, M. del R. y otros (2009). Trabajo Docente y Universidad Pública: políticas y subjetividades en los '90. Paraná, Entre Ríos: Fundación La Hendija.

Badano, M. y Díaz, R. (2010). Ficha de Cátedra: Análisis de Entrevista. Frases significativas. Seminario apoyo tesistas, Maestría en Trabajo Social, FTS, UNER. Versión digital: http:// www.fts.uner.edu.ar/catedras03/tfoi/mat_catedra/analisis_entrevistas_frases_significativas.pdf

Burín, M. (1987). Estudios sobre la subjetividad femenina. Mujer y Salud Mental. Buenos Aires: Grupo Editor Latinoamericano.

Bolívar Botía, A. (2002). ¿De nobis ipsis silemus? Epistemología de la Investigación Biográfico-Narrativa. Revista Electrónica de Investigación Educativa (REDIE), Vol. 4 (I). 
Castel, R. (1980). El orden psiquiátrico: la edad de oro del alienismo. Madrid: La Piqueta.

Castelnuovo, N. (2006). Frente al límite. Buenos Aires: Antropofagia.

Femenías, M. L. (2013). Violencias cotidianas (en las vidas de las mujeres), Prohistoria Ediciones, Rosario. - Col. Los ríos subterráneos.

Foucault, M. (2007). El Poder Psiquiátrico. Buenos Aires: Fondo de Cultura Económica.

García Calvente, M. del M., Mateo, I. y Eguiguren, A. (2009). El sistema informal de cuidados en clave de desigualdad. España: Escuela Andaluza de Salud Pública.

González Rey, F. L. (2011). El sujeto y la subjetividad en Psicología Social. Buenos Aires: Noveduc.

Glaser, B. y Strauss A. (1967). The discovery of grounded theory: strategies for qualitative research, New York, Aldine Publishing Company.

Goffman, E. (1998). Estigma. La identidad deteriorada. Buenos Aires: Amorrortu.

Idoyaga Molina, A. (Editora) (2007). Los caminos terapéuticos y los rostros de la diversidad. Buenos Aires: Editorial CAEA - IUNA.

Kornblit, A. L. (2004). Metodologías Cualitativas en Ciencias Sociales. Modelos y procedimientos de análisis. Argentina: Biblos.

Lindón, A. (1999). Narrativas autobiográficas, memorias y mitos: una aproximación a la acción social. Economía, Sociedad y Territorio, Vol. II, № 7. México.

Maxwell, J. (1996). Qualitative research desing. An Interactive Approach. California: George Mason University.

Rojas Ortíz, R. (2009). Género y Enfermedad Mental. Revista Margen, No 54 .

Shore, C. (2010). "La antropología y el estudio de la política pública: reflexiones sobre la "formulación" de las políticas" En: Antípoda No 10 enero-junio. Páginas 21-49.

Souza Minayo, M. C. (1997). El desafío del conocimiento. Investigación cualitativa en salud. Buenos Aires: Lugar Editorial. 